\title{
EEG Connectivity Analysis in Patients with Left and Right Hemispheric Mediobasal Meningiomas before and after the Therapeutic Radiation Exposure
}

\author{
M Yu Yarets ${ }^{1}$, A Yu Kuleva ${ }^{1}$, MV Galkin ${ }^{2}$, KD Vigasina ${ }^{1}$, YuV Strunina ${ }^{2}$, EV Sharova ${ }^{1 *}$ and OA Krotkova ${ }^{2}$ \\ ${ }^{1}$ Department of Nervous Activity and Neurophysiology, Institute of Higher Nervous Activity and Neurophysiology of RAS, Russia \\ ${ }^{2}$ Department of Neurosurgery, Federal State Autonomous Institution NN Burdenko National Medical Research Center of Neurosurgery of the Ministry of \\ Health of the Russian Federation, Russia
}

*Corresponding author: EV Sharova, Institute of Higher Nervous Activity and Received Date: November 05, 2019

Neurophysiology of RAS, Russia.

Published Date: November 12, 2019

Keywords: EEG; Coherence; Visual memorization; Radiation therapy; Meningioma

\section{Introduction}

Radiotherapy (RT) is the most effective way to treat cerebral tumors when surgical resection is not possible. The markers of RT effectiveness as well as the features of cerebral neurodynamics in the localization of the pathological process in the right and left hemispheres are practically not studied. Aims to investigate the dynamics of functional brain activity in patients with tumor lesions of mediobasal regions in the right or left hemispheres, positively affected by the radiotherapy.

\section{Materials and Methods}

The main observation group consisted of 10 patients with meningiomas of mediobasal regions (in 6 patients meningioma was in the right hemisphere, in 4 patients in the left hemisphere). The result of their stereotactic RT therapy was a reduction in tumor size (according to MRI). The condition of the patients was evaluated in dynamics: before the start of RT, immediately after its completion and after 6 months. At each control point, along with MRI study, the neuropsychological testing was performed according to the method of A.R. Luria [1], as well as an EEG study. The visual memorization was conducted [2] to identify and track the dynamics of executive functions' disorders (voluntary attention and its spatial distribution, visual working memory) observed in patients before treatment. The EEG study included 18-channel monopolar registration of biopotentials, using Nichon Kohden electroencephalograph (the reference - indifferent ear electrodes, bandpass - 0.3-35 Hz). Then the coherent spectral analysis was applied to the artifact-free EEG-segments (minimum - 60 seconds) of the resting state (eyes-closed and eyes-opened), as well as during visual memorization, using MBN-Neurokartograph software (Russia). The coherent quantitative EEG (CohEEG) parameters were calculated over the ranges of normal frequencies (delta-, theta-, alpha1-3, beta1-2) for all possible combinations of electrodes. The topography and reliability of CohEEG changes during visual memorization compared with the background with open eyes was determined using a specialized statistical software package based on the nonparametric Mann-Whitney test $[3,4]$. The control group included data from a similar study of 12 healthy subjects.

\section{Results}

The spatial changes of CohEEG in healthy people during visual memorization are shown in Figure 1I. The diffuse changes in connectivity were characteristic for them relative to the resting state with open eyes, pronounced to varying degrees in all frequency ranges. More generalized CohEEG attenuation was observed in the theta- and alpha- 1 bands. More local bilateral changes in alpha-3 and beta- 1 rhythms in symmetrical frontal regions were detected, associated with increased visual attention, according to the literature [5]. In all alpha subranges, there was the predominance of reactively altered CohEEG in the fronto-temporal regions of the right hemisphere (Figure 1). 
I

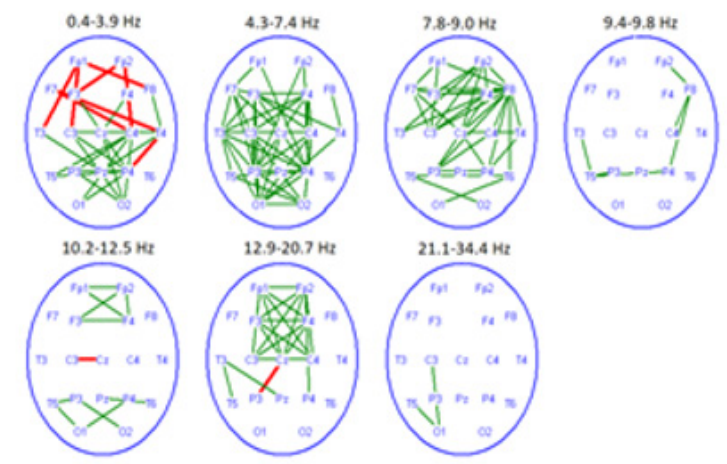

A

II

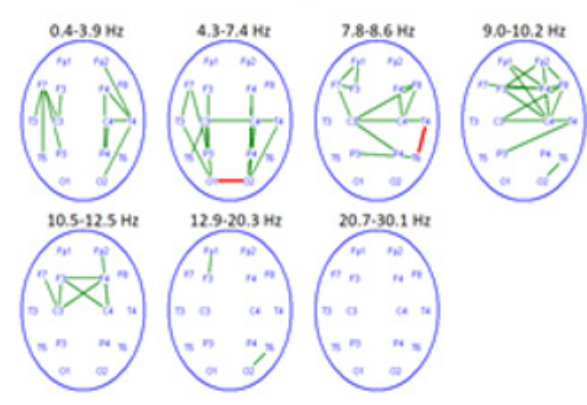

A

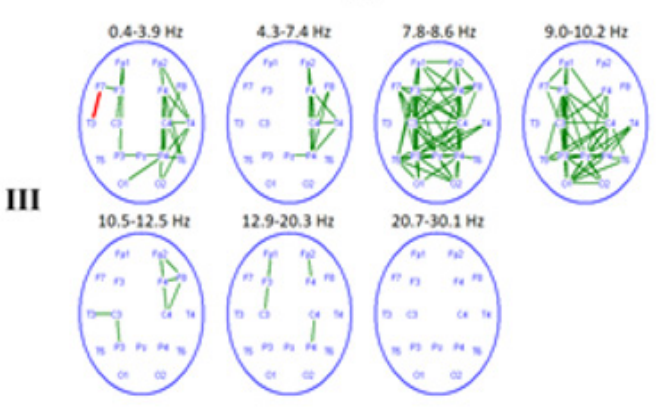

B
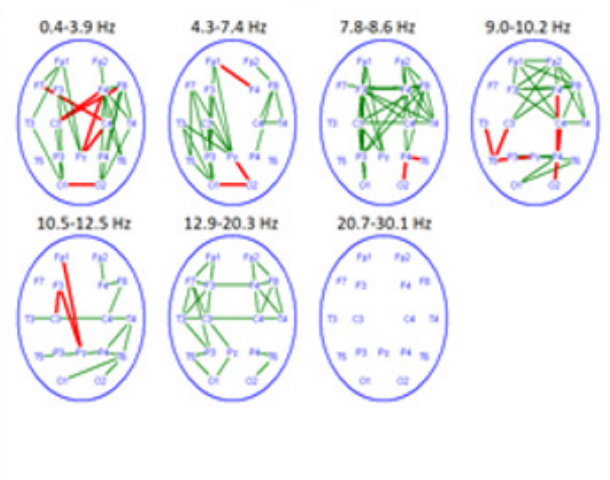

B

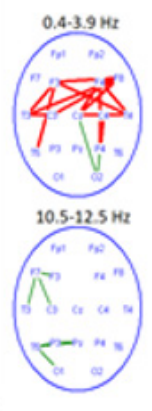

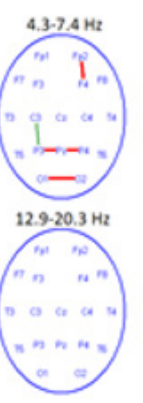

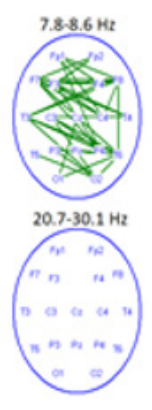

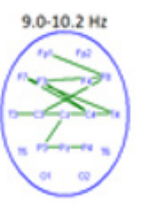

Figure 1: The changes of EEG coherency in different frequency bands during the visual memorization task compared to the background with open eyes. I - healthy subjects $(n=12)$, II - patients with left meningiomas in dynamics $(n=6)$, III - patients with right meningiomas in dynamics $(n=4)$; A) before RT, B) 6 months after RT. Red lines mark a significant coherence increase; green - a significant decrease (Mann-Whitney test, $p<0.05)$.

In patients with temporal mediobasal tumors before treatment, the EEG responses while visual memorization (Figure 1 IIA and III A) were as different as possible from the normal response, however, they had their own specificity due to lateralization of the lesion. The "depletion" of CohEEG changes was characteristic of both groups in the form of the decrease in the number of reactive bonds, especially interhemispheric, as well as the weakening or areactivity of the frontal cortical regions. In most frequency ranges (excepted beta), the weakening of CohEEG was more pronounced in patients with lesions of the left hemisphere (Figure 1 IIA), in particular, the weaking of CohEEG in alpha- 1 and beta- 1 ranges was observed. This corresponded to the violations during the oral/verbal reproduction of visual information, uncharacteristic for patients with right-sided lesion [6].
Six months after RT, EEG response while visual memorization in patients with lesions of the left hemisphere demonstrated the tendency to normalization (Figure 1 IIB): the increase in the number of reactively changing interhemispheric and left hemispheric CohEEG, especially in the alpha-1, alpha- 2 and beta1 ranges. This was accompanied by the decrease in the number of visual memory errors made by patients during neuropsychological testing. In patients with a tumor of the right temporal lobe (Figure 1 IIB), the features of EEG response normalization were manifested mainly in the reactive amplification of hemispheric CohEEG in the delta range, as well as the increase in reactivity in alpha- 2 in the right hemisphere. The weakening/areactivity of CohEEG rhythms of a higher frequency (alpha-3 and beta), especially in the anterior cortical regions, was greater in the right hemisphere. This was 
associated with the increase in the number of visual memorization errors during neuropsychological testing [7].

\section{Conclusion}

A comparative analysis of CohEEG changes while memorizing images of healthy subjects and patients with meningiomas of the mediobasal regions in the right and left temporal lobes before and after RT showed the correspondence of the spatial organization of the EEG response to the presence and severity of a cognitive defect in pathology according to neuropsychological testing. The brain reactivity features, connected with therapeutic radiation exposure were revealed, as well as the adaptive brain strategies to the effects of RT, associated with tumor lateralization. The improvement of EEG markers of visual memorization 6 months after RT corresponded to the direction of the substrate changes (the reduction in tumor size). This data indicates that the CohEEG spatial organization analysis is informative for assessing the effectiveness of radiotherapy in patients with cerebral pathology.

\section{Funding}

This work was supported by the grant of the Russian National Fund №17-15-01426.

\section{Acknowledgement}

None.

\section{Conflict of Interest}

The authors declare that the research was conducted in the absence of any commercial or financial relationships that could be construed as a potential conflict of interest.

\section{References}

1. Luria AR (1996) Higher cortical functions in man. (Originally published by Moscow University Press, 1962.) Translated by Basil Haigh. New York: Basic Books, USA.

2. OA Krotkova, MYu Kaverina, GV Danilov (2018) Eye Tracking and Interhemispheric Interaction in the Distribution of Spatial Attention. Human Physiology 44(2): 175-182.

3. Voronov VG, Shekutev GA, Grindel OM (2003) Paket programm dlya statisticheskogo sravnenia zapisei EEG. Materials of the international conference "Clinical neuroscience: neurophysiology, nevrology, neurosurgery", Crimea, Gurzuf, Ukraine, pp. 22-24

4. Sharova EV, Pogosbekyan EL, Korobkova EV, Zaitsev OS, Zakharova NE, et al. (2018) Interhemispheric connectivity and attention in patients with disorders of consciousness after severe traumatic brain injury. J Neurol Stroke 8(4): 245-253.

5. EV Sharova, GN Boldyreva, MA Kulikov, PE Volynskii, AV Kotenev, et al. (2009) EEG Correlates of the States of Visual and Auditory Attentionin Healthy Subjects. Human Physiology 35(1): 1-10.

6. Samuel E Day, Lia M Halasz (2017) Radiation therapy for WHO grade I meningioma. Chinese Clinical Oncology 6(Suppl 1): S4.

7. Apra C, Peyre M, Kalamarides M (2018) Current treatment options for meningioma. Expert Rev Neurother 18(3): 241-249. 